\title{
Fundamental Mechanism of Slow Crack Growth in Semi-Crystalline Polymers under a Constant Load
}

\author{
Norman Brown \\ Department of Material Science, University of Pennsylvania, Philadelphia, USA \\ Email: nbrown@seas.upenn.edu
}

How to cite this paper: Brown, N. (2019) Fundamental Mechanism of Slow Crack Growth in Semi-Crystalline Polymers under a Constant Load. Materials Sciences and Applications, 10, 721-731.

https://doi.org/10.4236/msa.2019.1011052

Received: September 16, 2019

Accepted: November 17, 2019

Published: November 20, 2019

Copyright (อ 2019 by author(s) and Scientific Research Publishing Inc. This work is licensed under the Creative Commons Attribution International License (CC BY 4.0).

http://creativecommons.org/licenses/by/4.0/

\begin{abstract}
The purpose is to quantitatively present in a single equation all the factors that affect the failure time by Slow Crack Growth (SCG) in a semi-crystalline polymer (SCP) under a constant load. The fundamental mechanism of fracture is displayed at the molecular level. The rate of fracturing is determined by the Eyring theory of thermal activation. The resulting equation includes the important molecular properties of therein, the length and density of the tie molecules. The underlying microfracture process is the unfolding of the chains in the crystal under the action of the tie molecules.
\end{abstract}

\section{Keywords}

Failure, Semi-Crystalline Polymer

\section{Introduction}

When a SCP structure is exposed to a sufficiently low constant tensile stress under plain strain conditions, it will ultimately fail by a brittle fracture process commonly called "slow crack growth" [SCG]. Polyethylene (PE), the most widely used SCP, was first used on a large scale in gas pipes in 1965 manufactured by the DuPont Company. The phenomenon of SCG has been subjected to extensive research throughout the world, because it is very important to know how long a gas pipeline will function before it fails. Much of the published research on PE was produced by $\mathrm{Lu}$ and Brown and coworkers at the University of Pennsylvania, as for example in reference [1], nevertheless, up to now, a completely quantitative expression taking into account all the factors that influence the failure time by SCG does not exist. It is important to know the aspects of molecular structure of the PE that influences SCG in order to help the resin producers im- 
prove their product. By knowing all the factors that influence SCG, the engineer can better design structures made of PE.

1) Failure by SCG only occurs when the structure is subjected to a tensile stress less than about 1/2 $Y$ under plain strain conditions where $Y$ is the yield point. When the stress is greater than $1 / 2 Y$, ductile failure is produced by the macroscopic accumulation of plastic strain. Below $1 / 2 \mathrm{Y}$, the amount of macroscopic plastic strain is negligible and brittle failure is produced by a crack. The failure in a pressurized pipe exhibits a dramatic difference between the modes of failure above and below 1/2 $Y$. The failure above 1/2 $Y$ consists of a bulge in the pipe whose dimensions are on the order of the pipe diameter. The failure below $1 / 2 \quad Y$ consists of a leak in the form of a slit whose width is a very small fraction of the wall thickness of the pipe.

2) The dependence of the failure time on stress at various temperatures in notched specimens is exhibited in Figure 1 (from Lu and Brown in Ref. [2]). The transition from ductile failure to SCG failure occurs in the jog where a crack is initiated at a point of stress concentration. At the stress above the jog the plastic strain blunts the stress concentrators. In the jog where the stress is reduced, the sharpness of the stress concentrator is increased to the point where the process of SCG takes over. Then the failure time, $t$, depends on the temperature. In agreement with the Arrhenius theory where $t=A \exp [Q / k T]$. The experimental value of Qranges from about 85 to $110 \mathrm{kj} / \mathrm{mol}$. for all PE.

3) Fracture always initiates at points of stress concentration. It is widely opined that practical structures contain points of stress concentration. The stress concentrators can take many forms such as inclusions, sharp corners, scratches and molding defects. Figure 2 shows the single edge notch stress concentrator that is frequently studied with the applied stress perpendicular to its length, a. This is called the $K_{\mathrm{I}}$ fracture mode.

The stress concentrator is conventionally described by the stress intensity, $K$, where $K=g a^{1 / 2} s, g$ is a geometric factor determined by the ratio of a with respect to the macroscopic dimension of the specimen and $s$ is the global stress. The subsequent analysis shows that $c$ must be included in the description of the stress concentrator order to completely account for all the factors that determine the time to produce failure under a constant load.

4) It is necessary to know the common morphology of all SCP at the molecular level. SCP are linear. Lamella crystals are formed that consists of folded molecules whose thickness may vary from about 40 to $100 \mathrm{~nm}$. The folded chains are parallel to the thickness direction. There is an amorphous boundary about 15 $\mathrm{nm}$ thick between the crystals. The microstructure is depicted in Figure 3(a). There are some molecules that connect one crystal to an adjoining crystal. These molecules are called "tie molecules" [TM]. If TM did not exist, PE would be a completely brittle material. Sometimes a molecule from one crystal can be entangled with a molecule from the adjacent crystal in the amorphous boundary. This entanglement can function as a TM. 


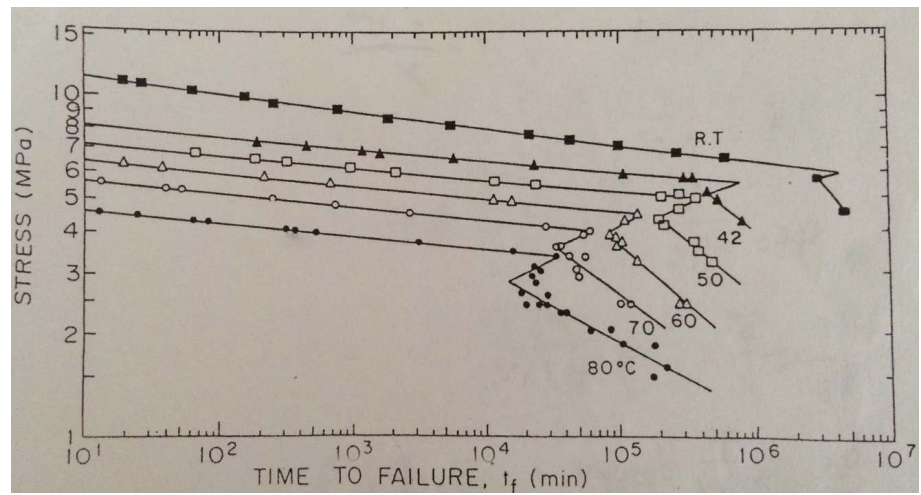

Figure 1. Stress vs. failure time various temperatures.

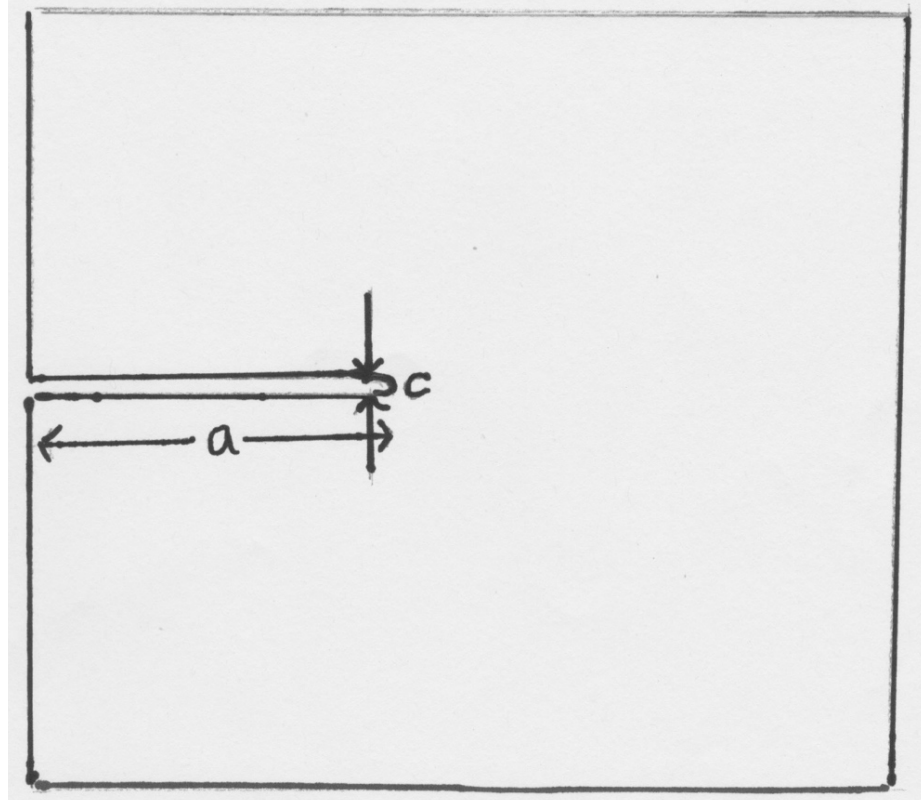

Figure 2. A representative stress concentrator; $c$ is the sharpness factor.

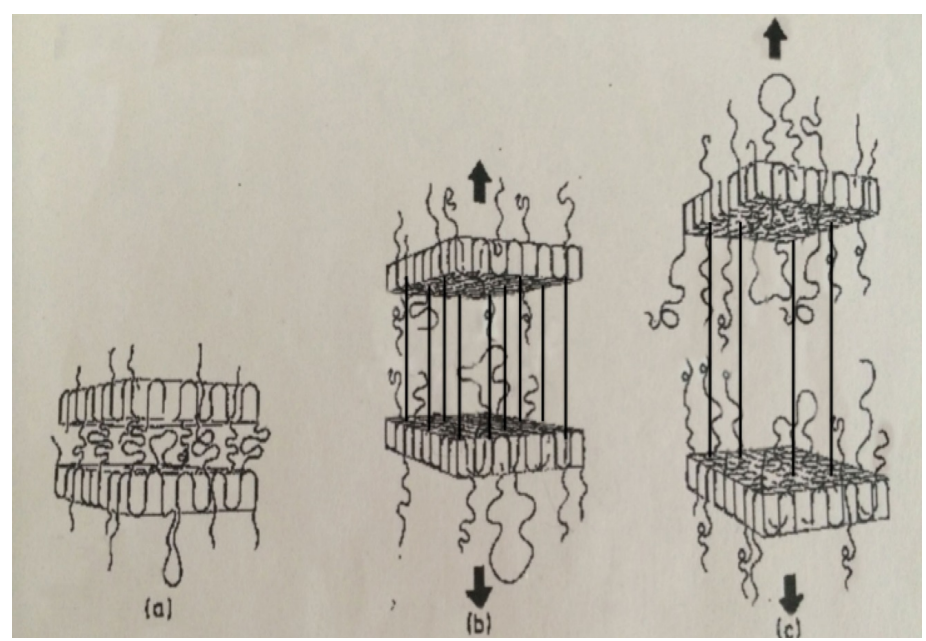

Figure 3. Morphology of a fibril. a: before loading; b: after loading; c: during incubation. 
5) When a tensile stress less than $1 / 2 Y$ is applied to a structure under plane strain conditions, a deformation zone is produced in the vicinity of the stress concentrators. It consists of coarse fibrils that are oriented in the direction of the applied stress (Figure 3(b)). Based on optical microscopic examinations, the deformation zone is illustrated in Figure 4. It was experimentally determined by Wang et al. [3] [4] that the stress on the boundary of the fibrils is equal to $Y$.

6) The crack opening displacement [COD] typically increases with time as shown in Figure 5, which is based on optical microscopic observations by Lu and Brown [5]. The original microstructure is converted to the deformation zone when the structure is loaded. The first fibril fractures at the time, $E$. It is important to note that the time to incubate fracture in the first fibril is always a significantly large fraction of the time for the complete failure in the structure. This is a general experimental observation. After the first fibril fractures, there is an acceleration in the rate of increase in the COD because the length of the stress concentrator increases.

The crack propagates by the successive fracture of the fibrils until the remaining ligament fails by yielding.

It is important to determine the stress in the first fibril because that is where fracture initiates. The geometry of the first fibril is illustrated in Figure 6. The boundary of the fibrils corresponds to the boundary of the deformation zone where the stress is $Y$. The area of the boundary of the deformation zone that is occupied by the first fibril is designated by $A_{o}$. The cross section area of the fibril is $A$. The length of the first fibril is the COD. The first fibril evolves from a volume $A_{o} c$, where $c$, the sharpness of the stress concentrator, is the thickness of the volume of material from which the first fibril evolves. Conservation of volume gives

$$
A_{o} c=A C O D
$$

The validity of Equation (1) is based on the assumption that $A$ is the average cross section area of the fibril and on the facts that the COD is very much greater than $c$ and also that the COD is independent of $c$.

Stress equilibrium requires

$$
A_{0} Y=A S
$$

where $S$ is the stress in the fibril. Combining Equations (1) and (2)

$$
S=Y \mathrm{COD} / \mathrm{c}
$$

It is important to know the morphology of the fibril at the molecular level. Based on the extensive research on the morphology of the fibril by Kieth et al. [6], the molecular morphology of a fibril is shown in Figure 3(b) where the lamellar crystals are joined together by TM and the thickness of the crystals is the same as in the preloaded state. Also the folded chains are oriented in the direction of the applied tensile stress. Also note in Figure 3(c) that the forces, $F$, that are exerted by the TM, must balance the force exerted by the stress $S$ in the crystal. Therefore 
s

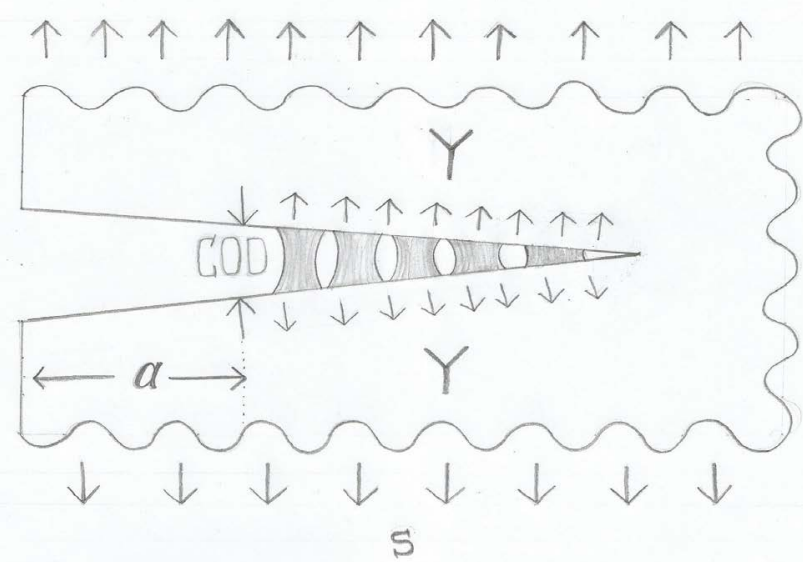

Figure 4. The deformation zone. $S$ is the global stress.

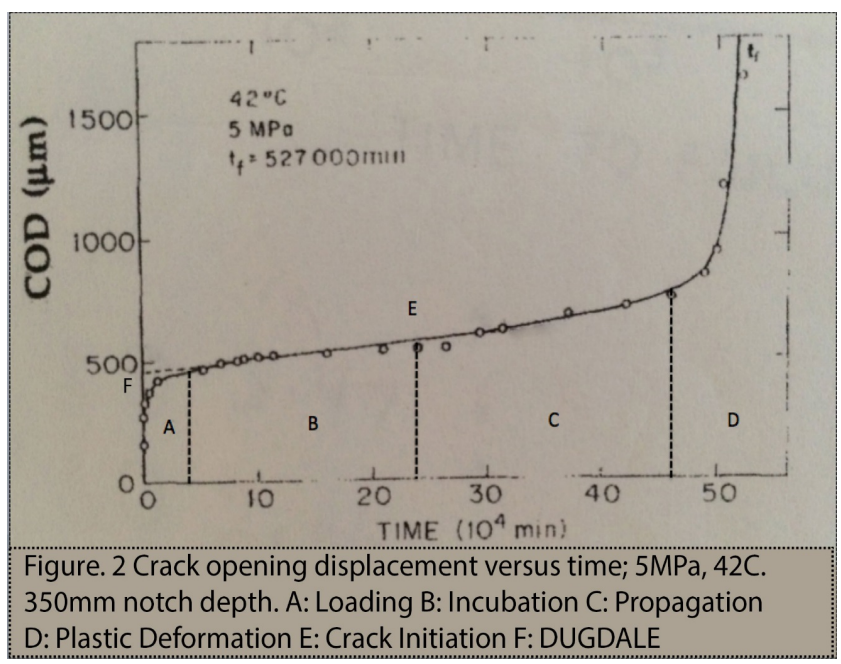

Figure 5. COD versus Time (by Qian and Lu in Reference [10]).

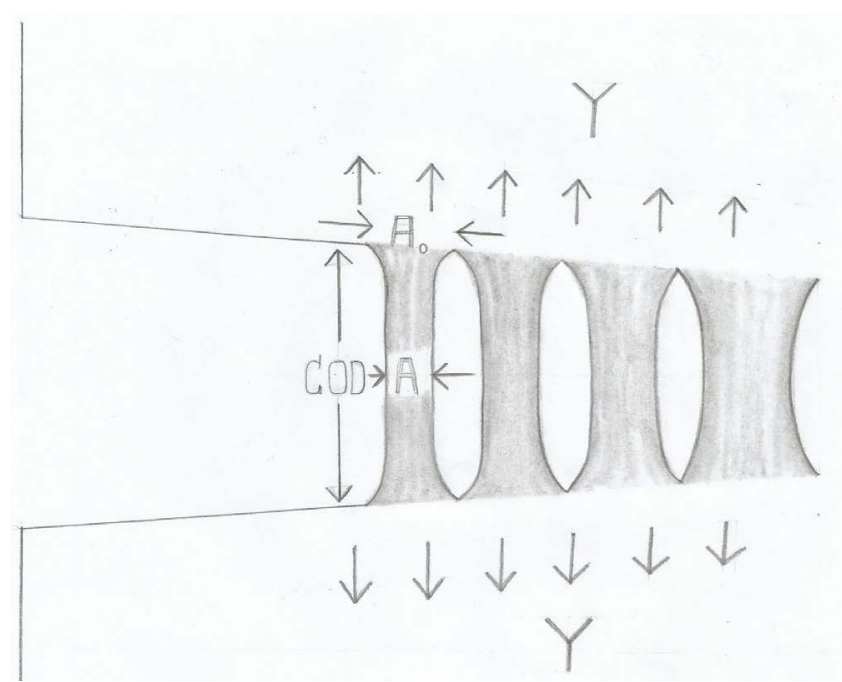

Figure 6. Basis for the analysis of the stress in the first fibril. 
where $A$ is the area on which $\mathrm{S}$ acts and $n$ is the number of TM. $A$ is also the area from which the TM emerges from the crystal. By definition, the density of TM is $\rho=n / A$. Combining (3) and (4) gives

$$
F=Y \mathrm{COD} / \rho c
$$

In order to better visualize the critical role that $F$ plays in the fracture process, the fundamental morphological unit of the fracture process is exhibited in Figure 7, where the TM with the force, $F$, is pulling on the folded chain and thereby unfolding the chains from the crystal. At the same time the length of the TM between the crystals increases as shown in Figure 3(c). When an entire $T$ is pulled out of the crystal, then TM is eliminated. That event represents the fundamental sub-microscopic fracture process during SCG. It naturally follows that the longer the length of a TM, the longer time it takes to eliminate the TM. When all of the TM in a cross section area of a fibril are pulled out of the crystal, the fibril fractures. The next question is, what is the rate at which the chains are unfolded?

\section{Rate of Unfolding the Chains}

The rate of unfolding the chains will be derived from the Eyring theory of thermal activation [7]. Follow the theory while focusing on Figure 7. The theory begins with the postulate that there is an energy barrier $Q$ that must be overcome in order that a portion of a chain is pulled out of the crystal. The value of $Q$ is the binding energy of a chain to the crystal. The force, $F$, exerts an energy, $F_{V}$, so that the barrier height is reduced to $Q-F_{V}$. $v$ is called the activation distance. $v$ is the displacement of a chain from its neighbor under the action of the force, $F . v$ is a small fraction of the distance between the chains. The probability of attaining the energy, $Q-F_{V}$, at the temperature, $T$ is given by the Maxwell-Boltzmann distribution $\exp [-(Q-F v) / k T]$. Visualize that the TM is undergoing thermal vibrations with a frequency $f$, which is the frequency at which the force, $F$. attacks the energy barrier. The frequency at which the barrier is overcome is $f \exp [-(Q-F v) / k T]$. Every time the barrier is overcome, a length $V$, of the TM is withdrawn from the crystal. $V$ is on the order of a fraction of a chain length. Thus, $r$, the rate of withdrawal of a TM from the crystal is given by

$$
r=V f \exp [-Q / k T] \exp [F v / k T]
$$

The value of $F$ from (5) will now be inserted into Equation (6).

$$
r=V f \exp [-Q / k T] \exp [Y \operatorname{COD} v / \rho c k T]
$$

In order to completely know all the parameters that determine $r$, the basis for determining the COD must be presented. The value for the COD was derived from a theory independently developed by Dugdale [8] and Rice [9] for the stress concentrator shown in Figure 2 for a material, whose mechanical behavior consists of linear elastic behavior up to the yield point $Y$ followed by no strain 


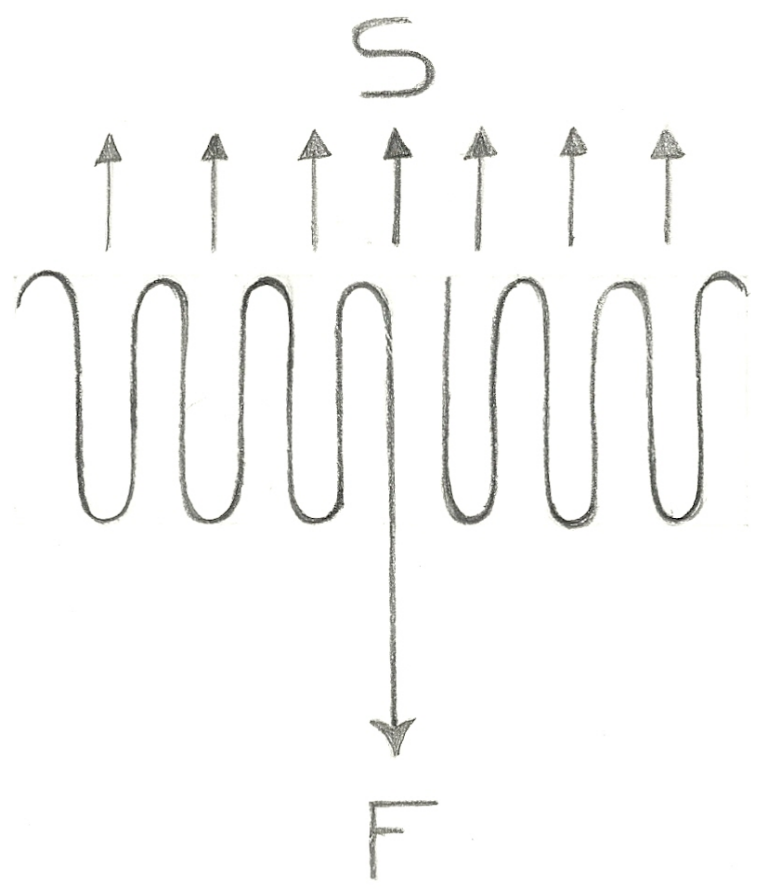

Figure 7. Fundamental process for unfolding Chains from the Crystal. $S$ is the stress in the Fibril. $F$ is the Force acting on a TM.

hardening after the yielding. Since PE only approximates the mechanical behavior of this material, the Dugdale-Rice formula is an approximation to the observed value of the COD. However, the experimental values of the COD vary in tune with the parameters of the Dugdale-Rice formula that

$$
C O D=K^{2} / Y E
$$

$K$, the stress intensity, is given by

$$
K=g s a^{1 / 2}
$$

$g$ is a geometric factor that depends on the geometry of the stress concentrators, $s$ is the applied stress and a is the length of the stress concentrator. $E$ is Youngs modulus.

The experimental values of the COD in PE as measured by Qian et al. [10] obey the following equation:

$$
\mathrm{COD}=B s^{n} a^{m}
$$

$B$ is a geometric factor like $g$. Depending on the resin, $n$ can go from about 3 4.5 and $\mathrm{m}$ is slightly greater than $1 / 2 n$. The experimental value of the COD approximates the Dugdale-Rice prediction. In order to emphasize the importance of the stress intensity, $K$, the Dugdale-Rice value of the COD is inserted in [7]. All the factors that determine the rate, $r$, at which a TM is pulled out of the crystal, are given in Equation (11).

$$
r=V f \exp [-Q / k T] \exp \left[K^{2} v / E \rho c k T\right]
$$


Fracture initiates in the structure when all the TM in a cross section area of the first fibril are pulled out. The time to fracture the first fibril depends on the length average $L_{z}$ of the longest molecules that contain TM. The time also depends on the rate, $r_{z}$ that is controlled by the density of TM, $\rho_{z}$, whose length is $L_{z}$. It is straight forward that the time to initiate fracture can be described by

$$
t=L_{z} / r_{z}
$$

Combining (11) and (12), the time to initiate fracture is

$$
t=\left(L_{z} / V f\right) \exp [Q / k T] \exp \left[-K^{2} v / E \rho_{z} c k T\right]
$$

After the first fibril fractures, the crack propagates by the successive fracture of the following fibrils. The time to fracture in a fibril is still governed by equation (13) with the exception that $\mathrm{K}$ increases because the length of the stress concentrator increases every time a fibril fractures. Thus, the curve of COD versus time in Figure 2 begins to accelerate after the first fibril fractures. Thus, the crack propagates until the stress in the remaining ligament of the structure increases to the point where final mode of failure occurs by plastic deformation.

\section{Experimental Support for the Theory}

Equation (13) predicts that $t$ is linearly related to the length of the [TM]. Huang and Brown [11] experimentally confirmed this prediction by experimentally exhibiting that $\mathrm{t}$ is linearly related the molecular weight, $M_{w}$. Equation (13) indicates that the ultimate limit on the time to failure depends on the ultimate length of the TM. Experiments with ultra high molecular PE were performed that lasted so long that the time of failure could not be measured under the available laboratory conditions.

The introduction of short chain branches [SCB] greatly increases the failure time in copolymers relative to a homopolymer because the density of TM is directly related to the concentration of the SCB. The reason for this relationship is very simple. Direct measurements show that over $90 \%$ of the SCB reside in the amorphous region which is the boundary between adjacent crystals (data from Dow Chemical Co. in private communication). Consequently the probability of forming a TM is directly related to the concentration of SCB.

Huang and Brown [12] showed that the log of the time to initiate fracture is a linear function of the concentration of SCB. This result is in agreement with Equation (13) because the concentration of SCB is directly proportional to the density of the TM.

Equation (13) predicts that the high end of the molecular weight distribution is a significant factor. Scholten and Rikjema [13] fractioned 5 resins and measured the concentration of SCB on each fraction. They measured the failure time, $t$, on each resin. They found that $t$ increased as the concentration of SCB on the highest molecular weight fraction increased. This result confirms Equation (13).

When the specimen is loaded as shown in Figure 3(c), the TM must be longer 
than the value of the COD predicted by Equation (8) in order form a bridge between the adjoining crystals. If the length of the TM is below this critical value, the specimen will fail immediately upon loading. Lu et al. [14] verified this prediction. They fractionated a copolymer into 5 parts whose molecular varied from about $10^{3}$ to $10^{5} \mathrm{gm} / \mathrm{mol}$. They found that the 3 parts whose molecular weight was less than $3000 \mathrm{gm} / \mathrm{mol}$ failed immediately upon loading. The other 2 parts with greater molecular weight had failure times on the order of $1000 \mathrm{hr}$, which is comparable to $t$ for the whole resin.

Huang and Brown [11] measured the failure time as a function of molecular weight. The extrapolation of the molecular weight to $t=0$ was about 30,000 $\mathrm{gm} / \mathrm{mol}$. This result is consistent with the above results by Lu et al. [14].

Industry developed resins with a bimodal molecular weight distribution with no SCB on the low molecular weight side of the distribution. If the low molecular weight molecules contained SCB these SCB would be wasted as soon as the structure is loaded. For some application there is a limit on the concentration of SCB because the yield point decreases as the concentration of SCB is increased. When designing a structure, the engineer often optimizes the performance by making a compromise between the yield strength and failure time by SCG.

The inclusion of the notch sharpness factor, $c$, in Equation (13) is supported by the experimental observation by Lu et al. [15] that the notches produced by an ordinary commercial razor results in a failure time that is $10 \%$ longer than notches produced by specially sharpened commercial razor blades. The sharpness of the notch, $c$, is also determined by the speed at which the razor blade produces the notch. It was observed by Lu et al. [16] that above a critical speed the time to failure increased because the speed of notching affects the sharpness.

Lagaron et al. [16] measured the force, $F$, on a TM by a combination of optical microscopy and micro Raman spectroscopy. They observed the rate of SCG in several resins and found that $F$ increased as the rate of extension of the fibrils increased. This result conforms with Equation (6).

When a SCG test is measured in an Igepal environment, there is a significant decrease in the failure time (Paper by Ward et al. [17]). This effect modifies Equation (13) by reducing the value of $Q$, the bonding energy of the chains to the crystal, and the remaining terms in Equation (13) are unaltered. Consequently experiments show that the ranking of resins with respect to their failure time is the same whether the test is in air or in any plasticizing agent such as Igepal.

It is also interesting to know why the activation energy is about $100 \mathrm{kj} / \mathrm{mol}=1$ ev. The activation energy, $Q-F_{V}$, is largely determined by $Q$, the bonding energy between a folded chain and the crystal. Although the bonding is based on the Van der Waals bond whose energy is about $0.01 \mathrm{ev}, Q$ is about $1 \mathrm{ev}$ because it is an integration of all the bonds that surround a chain.

It is also interesting to note that whether the chains are regularly or irregularly folded the basic theory is not changed. The only difference is that the $Q$ for irregularly folded chains is expected to be less than that for regularly folded chains. 


\section{Summary and Conclusions}

1) The rate of SCG is thermally activated as described by the Eyring theory.

2) Equation (13) contains all the quantities that determine the time for the initiation of fracture by SCG in a semi-crystalline polymer. This is the first time that the process of SCG has been completely described by an equation.

3) The morphological features of the resin that determine the failure time are $L_{z}$, the length of the TM, whose molecular weight is $M_{z}$, and the density of the TM, $\rho_{z}$ whose molecular weight is $M_{z}$. These morphological features are controlled by the conditions under which the resin was polymerized.

4) The fundamental mechanism of SCG is the unfolding of the chains in the crystal under the action of the TM.

5) The time to initiate fracture in the first fibril occupies a major part of the total time for failure by SCG.

6) The density of the TM is proportional to the concentration of SCB because over $90 \%$ of the SCB are located in the boundary between the crystals.

\section{Acknowledgements}

Dr. Campbell Laird edited the manuscript.

\section{Conflicts of Interest}

The author declares no conflicts of interest regarding the publication of this paper.

\section{References}

[1] Lu, X., Qian, R. and Brown, N. (1991) Notchology-The Effect of the Notching Method on the Slow Crack Growth Failure in a Tough Polyethylene. Journal of Materials Science, 264, 881-888. https://doi.org/10.1007/BF00576763

[2] Lu, X., Qian, R. and Brown, N. (1990) The Transition from Ductile to Slow Crack Growth Failure in a Copolymer of Polyethylene. Journal of Materials Science, 25, 411-416. https://doi.org/10.1007/BF00714048

[3] Wang, X., Fager, L.O. and Brown, N. (1989) The Stress Distribution on the Boundary of Crazes in Polyethylene by the Direct and Semi-Theoretical Method. Polymer, 30, 453-460. https://doi.org/10.1016/0032-3861(89)90013-X

[4] Wang, X. and Brown, N. (1989) The Stress and Strain Fields in the Neighbourhood of a Notch in Polyethylene. Polymer, 30, 1456-1461. https://doi.org/10.1016/0032-3861(89)90215-2

[5] Lu, X. and Brown, N. (1986) The Relationship of the Initiation Stage to the Rate of Slow Crack Growth in Linear Polyethylene. Journal of Materials Science, 21, 2423-2429. https://doi.org/10.1007/BF01114287

[6] Kieth, H.D., Padden, F.J. and Vadimski, R.G. (1966) Intercrystalline Links in Polyethylene Crystallized from the Melt. Journal of Polymer Science Part A-2: Polymer Physics, 4, 267-281. https://doi.org/10.1002/pol.1966.160040208

[7] Eyring, H. (1941) Theory of Rate Process. McGraw Hill, New York.

[8] Dugdale, D.S. (1960) Yielding of Steel Sheets Containing Slits. Journal of the Mechanics and Physics of Solids, 18, 100-104. 
https://doi.org/10.1016/0022-5096(60)90013-2

[9] Rice, J.R. (1967) ARPA SD Report E39. Department of Defence.

[10] Qian, R., Lu, X. and Brown, N. (1989) Investigating the Existence of a Threshold Stress Intensity for Slow Crack Growth in High-Density Polyethylene. Journal of Materials Science, 24, 2467-2472. https://doi.org/10.1007/BF01174513

[11] Huang, Y. and Brown, N. (1988) The Effect of Molecular Weight on Slow Crack Growth in Linear Polyethylene Homopolymers. Journal of Materials Science, 23, 3648-3655. https://doi.org/10.1007/BF00540508

[12] Huang, Y. and Brown, N. (1991) Dependence of Slow Crack Growth in Polyethylene on Butyl Branch Density: Morphology and Theory. Journal of Materials Science, 29, 129.

[13] Scholten, F.L. and Rijkema, H.G. (1988) The Effect of Morphology on Slow Crack Growth in Polyethylene. Proceedings of the Plastics \& Rubber Institute of London, Paper c2/4.

[14] Lu, X., Ishikawa, N. and Brown, N. (1996) The Critical Molecular Weight for Resisting Slow Crack Growth in Polyethylene. Journal of Polymer Science Part B: Polymer Physics, 34, 1809-1813.

[15] Lu, X., Qian, R. and Brown, N. (1991) Discontinuous Crack Growth in Polyethylene Under a Constant Stress. Journal of Materials Science, 29, 881.

[16] Lagaron, J., Cappacio, G., Rose, L. and Kip, B. (2000) Craze Morphology and Molecular Orientation in the Slow Crack Growth Failure of Polyethylene. Journal of Applied Polymer Science, 77, 283-296. https://doi.org/10.1002/(SICI)1097-4628(20000711)77:2<283::AID-APP5>3.0.CO;2-0

[17] Ward, A., Lu, X., Huang, Y. and Brown, N. (1991) Accelerated Test for Evaluating Slow Crack in Polyethylene Co polymers in Nepali and Air. Macromolecular Chemistry, 41, 2172. 